\title{
Application of the Mattis-Bardeen theory in strongly disordered superconductors
}

\author{
G. Seibold, ${ }^{1}$ L. Benfatto, ${ }^{2}$ and C. Castellani ${ }^{2}$ \\ ${ }^{1}$ Institut für Physik, BTU Cottbus-Senftenberg, P.O. Box 101344, 03013 Cottbus, Germany \\ ${ }^{2}$ ISC-CNR and Department of Physics, University of Rome "La Sapienza", Piazzale Aldo Moro 5, 00185, Rome, Italy
}

(Received 10 February 2017; revised manuscript received 17 August 2017; published 11 October 2017)

\begin{abstract}
The low-energy optical conductivity of conventional superconductors is usually well described by MattisBardeen (MB) theory, which predicts the onset of absorption above an energy corresponding to twice the superconducing (SC) gap parameter $\Delta$. Recent experiments on strongly disordered superconductors have challenged the application of the MB formulas due to the occurrence of additional spectral weight at low energies below $2 \Delta$. Here we identify three crucial items that have to be included in the analysis of optical-conductivity data for these systems: (a) the correct identification of the optical threshold in the Mattis-Bardeen theory and its relation with the gap value extracted from the measured density of states, (b) the gauge-invariant evaluation of the current-current response function needed to account for the optical absorption by SC collective modes, and (c) the inclusion into the MB formula of the energy dependence of the density of states present already above $T_{c}$. By computing the optical conductivity in the disordered attractive Hubbard model, we analyze the relevance of all these items, and we provide a compelling scheme for the analysis and interpretation of the optical data in real materials.
\end{abstract}

DOI: 10.1103/PhysRevB.96.144507

\section{INTRODUCTION}

The Bardeen-Cooper-Schrieffer (BCS) theory of superconductivity [1] is probably one of the most successful examples in condensed matter of a microscopic approach able to describe a phase transition via the modification of the quasiparticle spectrum. In particular, the opening of a superconducting (SC) gap $\Delta$ below $T_{c}$, along with the emergence of a purely diamagnetic response (Meissner effect), are the basic ingredients required to interpret the thermodynamic and transport properties of conventional superconductors. However, to understand the finite-frequency optical response in the superconducting state, additional effects due to the presence of disorder must be included as well. The straightforward extension of BCS theory in the presence of nonmagnetic impurities was indeed developed soon after by Mattis and Bardeen (MB) [2] and independently by Abrikosov and Gorkov [3]. In this case the single-particle excitations are modified not only by the pairing, but also by the broadening $\Gamma$ of the energy levels due to scattering by impurities, which is present already above $T_{c}$. The consequences for the optical absorption below $T_{c}$, i.e., the real part of the optical conductivity $\sigma_{1}(\omega)$, have been worked out explicitly in the milestone MB work [2]. While in the clean case all carriers (density $n$ ) contribute to the superfluid $\delta$-like response at zero frequency, in the dirty limit only a fraction $n_{s} \sim n(\Delta / \Gamma)$ of the carriers condenses in the superfluid state. By sum-rule conservation the reduction of $n_{s}$ has a direct counterpart in the emergence of a finite-frequency optical absorption. Indeed, the MB model predicts the onset of absorption at $T=0$ above the superconducting gap $2 \Delta$, while for finite temperatures $T<T_{c}$ an additional quasiparticle contribution appears also below $2 \Delta$. In the intermediate disorder regime $\Gamma \sim \Delta$ the analytical MB formula does not apply, but a finite-frequency absorption above $2 \Delta$ still survives [4].

The MB theory successfully explains the microwave data in moderately disordered superconductors, as early experiments in indium and tin films demonstrated $[5,6]$. Later on it has been proven that the MB scheme can be extended to include also strong-coupling effects [7], as observed, e.g., in lead [8], or a temperature-dependent scattering rate of the residual (normal) quasiparticle excitations below $T_{c}$, as noticed in the analysis of Al films [9]. Only recently, studies on strongly disordered films of conventional superconductors [10-16], granular superconductors [17,18], and even cuprate superconductors [19] have revealed systematic deviations from the MB paradigm in the form of an extra subgap absorption, that fairly exceeds the quasiparticle contribution of the MB theory. In the case of homogeneously disordered films [10-15], the identification of this subgap contribution also relies on the simultaneous estimate of the pairing energy scale from the tunneling spectra, which by itself is a nontrivial issue. Indeed, tunneling measurements performed by several groups [20-27] revealed that in strongly disordered films of $\mathrm{NbN}, \mathrm{InO}_{x}$, and $\mathrm{TiN}$, where disorder induces a direct superconductor-to-insulator transition (SIT), the density of states (DOS) shows significant deviations from the usual BCS form, as summarized in Figs. 1(a) and 1(b). More specifically, while BCS theory at $T \ll T_{c}$ predicts a square-root divergence of the DOS at $\pm \Delta$, and the total suppression of the available states below the gap, the measurements reveal strongly suppressed coherence peaks at an energy $E_{\text {peak }}$ and a finite DOS below it, with tails extending up to a somehow lower energy $E_{\text {gap }}$. In addition, the local density of states probed by means of scanning tunneling spectroscopy (STS) shows that these features vary on the nanometer scale, leading to an inhomogeneous pattern of the local SC properties even in the presence of structurally homogeneous disorder $[21,25,26]$. This emergent granularity bears also nontrivial space correlations [21,25,28], with glassy features and quantum-percolation effects that have been interpreted theoretically as an outcome of the competition between pair hopping and localization at the verge of the SIT [28-35]. In this scenario, as the SIT is approached the pairing scale, $E_{\text {gap }}$ stays finite even when the average SC order parameter $\Delta \sim T_{c}$ softens, in agreement with tunneling observation $[21,22,25]$. All these features, which cannot be 

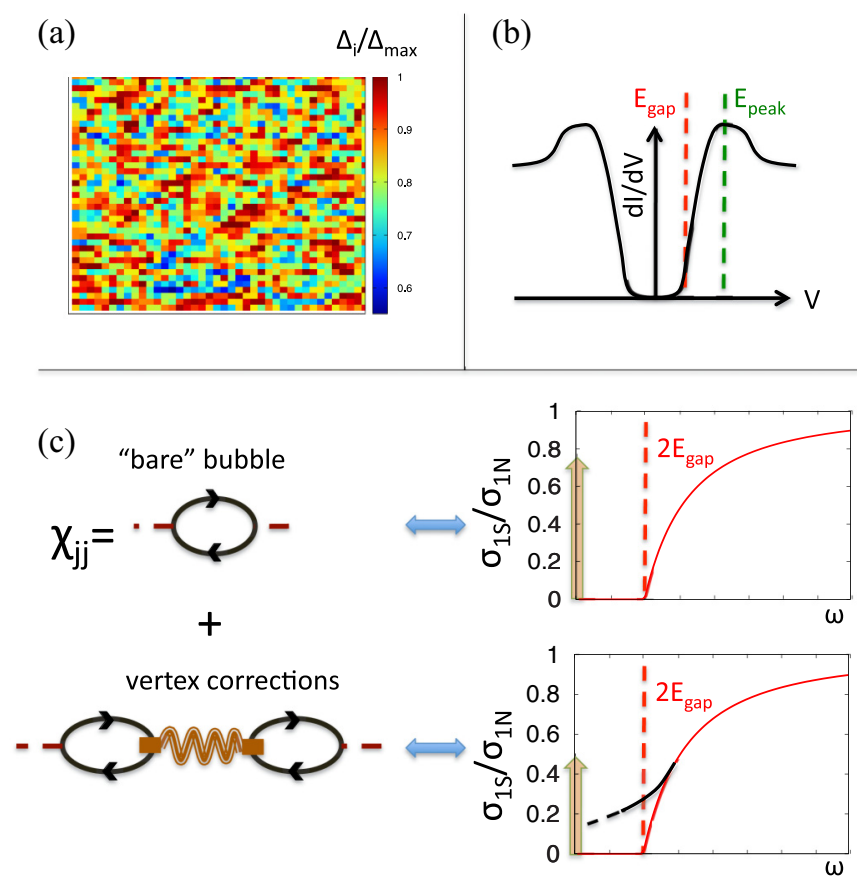

FIG. 1. Summary of the main spectral properties of strongly disordered superconductors. (a) Disorder induces a strong variation of the local SC order parameter $\Delta_{i}$, which is captured already at the level of the Bogoliubov-de Gennes solutions for the disordered attractive Hubbard model [28,29,32,33]. Experimentally, the variations occur as fluctuations of the coherence-peak height in the local DOS probed by STS $[23,25,28]$. (b) Sketch of the typical average tunneling DOS, as found numerically (see Fig. 2 below) and experimentally [20-27]. The typical SC coherence peaks are strongly suppressed in intensity, and they are located at an energy scale $E_{\text {peak }}$ somehow larger than the minimum excitation energy $E_{\text {gap }}$. (c) Optical conductivity of disordered SC. Here the solid lines denote the SC Nambu Green function, the dashed lines the e.m. field, and the wavy line the collective modes computed at RPA level, which build up the vertex corrections. The BCS approximation is equivalent to compute the "bare bubble" diagram, which accounts only for the breaking of Cooper pairs by the incoming e.m. field. Even when the SC order parameter is inhomogeneous, as in panel (a), it leads to an optical conductivity with a hard threshold at $2 E_{\text {gap }}$, that reproduces for intermediate disorder the usual Mattis-Bardeen results. The RPA vertex corrections account for the exchange of collective excitations between the Cooper pairs broken apart by the e.m. field. When these are included the optical conductivity acquires an extra contribution which piles up below $2 E_{\text {gap }}$, stealing spectral weight from the superfluid peak at zero frequency, denoted here by an arrow. The black dashed line indicates that the precise form of $\sigma_{1 s}$ as $\omega \rightarrow 0$ depends on the disorder level.

captured with simple phenomenological models, as, e.g., the Dynes formula [36], raise the nontrivial issue of the correct identification from the DOS of the energy scale to be compared with the measured optical absorption threshold.

From the theoretical point of view, the failure of the MB theory at strong disorder is not unexpected, since the MB theory describes only the effect of disorder on the quasiparticle response, but it neglects the contribution of collective modes. This distinction relies on the usual diagrammatic expansion of the current-current correlation function $\chi_{j j}$, which enters the electromagnetic (e.m.) kernel within the Kubo formalism (see Sec. II below for further details). At leading order, $\chi_{j j}$ is given by a bubble diagram which represents the excitation of a particle-hole pair by the incoming e.m. field; see Fig. 1(c). At BCS level one just computes this "bare bubble" diagram by taking into account the modification of the electronic single-particle spectrum and the presence of anomalous electronic averages in the SC state, leading to all the well-known predictions of the BCS theory for the transport properties [37]. On the other hand, below $T_{c}$ also collective SC excitations are possible, connected to the amplitude and phase fluctuations of the SC order parameter around the BCS mean-field equilibrium value. The former one is a massive mode, also referred to as Higgs mode due to the analogy with the particle of the standard model [38], while the second one is massless at long wavelength, since it represents the Goldstone mode of the U(1) gauge symmetry breaking in the SC state [39]. The effect of collective modes can be included within a gauge-invariant (GI) random-phase-approximation (RPA) by computing the vertex corrections to the current-current correlation function, that corresponds to account for all the intermediate processes between the particle-hole pairs excited by the e.m. field before than they recombine; see Fig. 1(c). In the clean case and for transverse fields both the Higgs and phase modes are decoupled from the current at long wavelength, even though the phase modes are crucial to restore the full gauge invariance of the longitudinal response function [37,40-42]. Thus, as long as one focuses on the transverse e.m. response the BCS approximation successfully explains the physics of conventional clean superconductors, even though it is not gauge invariant.

In the presence of disorder the computation of the SC e.m. response is more complicated. First, disorder affects the single-particle excitations. This effect is already present at the level of the bare-bubble approximation, and it is described by the MB theory with some additional approximations, as, e.g., a constant DOS of the electrons in the normal state and a clean BCS-like DOS in the SC state. As we mentioned before, this is not always the case in disordered films, where the DOS at the Fermi level is usually suppressed already above $T_{c}[14,21,22]$ and the BCS coherence peaks at $E_{\text {peak }}$ are smeared out in the SC state, with tails below it [20-22,25-27]. Such a modification of the DOS can be reproduced by strong on-site disorder [29,32], but also, e.g., within the Larkin-Ovchinnikov model [43], which implements a spatially varying depairing mechanism, via a position-dependent coupling constant (for a review $\mathrm{cf}$., e.g., Ref. [44]). The latter case is similar to what happens for magnetic impurities [45-47], which create in-gap bound states down to a scale $E_{\text {gap }}$ slightly below $E_{\text {peak }}$. In contrast the nonmagnetic impurities, considered in this manuscript, do not break time-reversal symmetry and therefore do not create bound states, but alter the SC gap parameter in their vicinity. How these modifications of the DOS reflect in the optical spectrum is still a quite unexplored issue. For the case of magnetic impurities it has been shown $[45,46]$ that the optical spectrum is well reproduced by the usual MB theory, expect for a tail which develops below $2 E_{\text {peak }}$ due to in-gap states. Recently, a similar result has been obtained in Ref. [14], where the MB theory has been modified with a DOS adopted 
from the Larkin-Ovchinnikov model [43]. In this view the MB response is argued to have still an optical threshold at $2 E_{\text {peak }}$, with absorption tails below it due to the existence of in-gap states down to $2 E_{\text {gap }}$. However, this analysis does not provide a good fit of the data, since it appears to underestimate the absorption in a large-frequency range starting right above the optical threshold.

In addition to the single-particle effects, disorder affects also the optical observability of the collective modes, making them possible candidates to explain the experimental anomalies. So far, two main proposals have been put forward. From one side, it has been emphasized [48] that disorder mixes the response at finite and zero momentum, making the contribution of the RPA vertex corrections finite even for the physical transverse response functions. While it has been clearly proven [48] that collective modes can then lead to additional absorption with respect to the BCS result, it has not been clarified yet where this extra dissipation occurs with respect to the typical energies scale $E_{\text {gap }}, E_{\text {peak }}$ identified by STM. A second proposal, put recently forward by Refs. $[13,49]$, focuses instead on the possibility that the amplitude Higgs mode contributes to the extra absorption via optical processes beyond RPA level, present in principle already for the clean system $[49,50]$. In this case the crucial role of disorder should be to change the nature itself of the mode, moving the Higgs resonance below the quasiparticle continuum at $2 E_{\text {gap }}$, restoring thus its relativistic dynamics [51,52]. However, this interpretation has been recently questioned in Ref. [52], where it has been shown that a strong overdamping of the Higgs mode persists even at strong disorder.

In this paper we present a detailed investigation of the optical properties of disordered superconductors with the aim to clarify all the possible mechanisms leading to significant deviations from the MB paradigm, including the variation of the DOS on the scale of the SC gap and the relevance of collective modes at low frequencies. Moreover, we aim to provide a general scheme to interpret current experiments in terms of an extended MB formula. We model the system by means of the attractive Hubbard model with on-site disorder, which has been already shown [28-30,32-35,52] to reproduce many of the unconventional features observed experimentally in strongly disordered thin films as, e.g., the granular structure of the SC state. In this context we refer to Refs. [53,54] for a review on experimental and theoretical aspects of disordered superconductors that also include systems where the transition between insulator and SC occurs via an intermediate metallic regime and which are not in the focus of our investigations.

By solving the Bogoliubov-de Gennes (BdG) equations we can describe the mean-field ground state in the presence of disorder. The optical conductivity is then computed both at the BCS level, i.e., as the bare-bubble response, and by adding the RPA vertex corrections. The computation of the BCS response allows us to investigate how the inhomogeneity of the SC properties triggered by strong disorder modifies the mean-field response with respect to the predictions of the MB theory, and to identify the relevant energy scales for the optical absorption. In particular, we investigate the role of two separate effects observed in the quasiparticle DOS: (i) the smearing of the SC peak due to inhomogeneity of the local SC order parameter $\Delta_{i}$; (ii) the persistence of a low-energy suppression of the DOS (pseudogap) above $T_{c}$. As far as item (i) is concerned, we show that inhomogeneity not only smears out the coherence peak at $E_{\text {peak }}$, but it also induces low-energy tails down to a quasiparticle gap $E_{\text {gap }}$ smaller than $E_{\text {peak }}$. On the other hand, the BCS optical response still displays a sharp optical absorption clearly located at $2 E_{\text {gap }}$, and not at $2 E_{\text {peak }}$. This result has to be contrasted with the case of magnetic impurities $[45,46]$ or spatially varying coupling constant [14], where the optical spectrum develops a tail below $2 E_{\text {peak }}$, due to the fact that the separation between $E_{\text {peak }}$ and $E_{\text {gap }}$ is due to in-gap bound states induced by a pair-breaking mechanism. For what concerns item (ii), i.e., the presence of a suppression in the normal-state DOS, we show that it can lead to observable deviations from the usual MB theory, which assumes a constant DOS. To account for this latter effect we propose a modification of the MB formula that compares very well with the explicit theoretical calculations of the BCS response and with the experimental data of Ref. [14] at intermediate disorder. Finally, when we add the contribution of collective modes, we observe that the modifications to the BCS response manifest mainly as an extra spectral weight below $2 E_{\text {gap }}$. While at intermediate disorder this extra absorption appears as a smearing of the BCS threshold at $2 E_{\text {gap }}$, at strong disorder the response of the collective modes resembles a peak at low frequency, which removes a considerable amount of spectral weight from the superfluid response [33].

The plan of the paper is the following. In Sec. II we introduce the model and the basic definitions. In Sec. III we analyze the DOS of the system in the presence of disorder. With respect to previous work [29,32], we clarify how disorder induces the formation of low-energy tails below the peak energy scale $E_{\text {peak }}$, leading to a lower value for the quasiparticle excitation threshold $E_{\text {gap }}$. In Sec. IV we report the results for the optical conductivity, and we demonstrate that while the BCS response still displays a hard threshold at $2 E_{\text {gap }}$, the collective modes induce additional low energy spectral weight. In Sec. IV we analyze quantitatively the BCS response by providing a generalization of the Mattis-Bardeen theory to reproduce the shape of the normal-state DOS, which is strongly frequency-dependent on the scale of the gap energy. We first demonstrate the validity of this generalization in the framework of the Hubbard model and then we show in Sec. VI that the theory gives also an excellent description of experimental data at intermediate disorder as exemplified by the optical measurements from Ref. [14]. Finally, we compare our GI calculations with the experimental results on the most disordered films, where a substantial subgap absorption has been reported. Section VII contains our summary and the concluding remarks.

\section{MODEL AND FORMALISM}

Our considerations are based on the attractive Hubbard model $(U<0)$ with local disorder,

$$
H=\sum_{i j \sigma} t_{i j} c_{i \sigma}^{\dagger} c_{j \sigma}+U \sum_{i} n_{i \uparrow} n_{i \downarrow}+\sum_{i \sigma} V_{i} n_{i \sigma},
$$


which we solve in mean-field using the BdG transformation,

$$
c_{i \sigma}=\sum_{k}\left[u_{i}(k) \gamma_{k, \sigma}-\sigma v_{i}^{*}(k) \gamma_{k,-\sigma}^{\dagger}\right],
$$

where the $u_{i}(k)$ and $v_{i}(k)$ variables are solution of the equations

$$
\begin{aligned}
\omega_{k} u_{i}(k)= & \sum_{j} t_{i j} u_{j}(k)+\left[V_{i}-\frac{|U|}{2}\left\langle n_{i}\right\rangle-\mu\right] u_{i}(k) \\
& +\Delta_{i} v_{i}(k), \\
\omega_{k} v_{i}(k)= & -\sum_{j} t_{i j}^{*} v_{j}(k)-\left[V_{i}-\frac{|U|}{2}\left\langle n_{i}\right\rangle-\mu\right] u_{i}(k) \\
& +\Delta_{i}^{*} u_{i}(k) .
\end{aligned}
$$

Here $\Delta_{i}$ and $\left\langle n_{i}\right\rangle$ represent the local SC order parameter and the local density, respectively, defined by the self-consistent equations

$$
\begin{gathered}
\Delta_{i}=|U| \sum_{k} u_{i}(k) v_{i}^{*}(k), \\
\left\langle n_{i}\right\rangle=2 \sum_{k}\left|v_{i}(k)\right|^{2} .
\end{gathered}
$$

The full sets of Eqs. (3)-(6) are solved self-consistently giving the configuration of the mean-field ground state in the presence of disorder. For simplicity only nearest-neighbor hopping $t_{i j}=$ $-t$ is considered in this work. The disorder variables $V_{i}$ are taken from a flat, normalized distribution ranging from $-V_{0}$ to $+V_{0}$. Moreover, we will take units $\hbar=c=1$ in all the paper, unless explicitly stated.

For $V_{0}=0$ and values of $|U| / t \leqslant 2$, which are considered in the present paper, the system is a standard BCS superconductor. However, upon including a sizable $V_{0} / t$ it has been shown in several works before [28-30,32-35], that the model Eq. (1) describes already at mean-field level many features observed experimentally in thin films of conventional SC near the SIT. In particular, the local $\mathrm{SC}$ order parameter $\Delta_{i}$ is strongly suppressed with increasing $V_{0} / t$, while the quasiparticle gap $E_{\text {gap }}$ (i.e., the minimum value of the quasiparticle energies $\omega_{k}$ ) saturates, indicating the formation of local, incoherent pairs. In addition, $\Delta_{i}$ segregates spontaneously by forming good SC regions embedded in a poorly SC background. Here we investigate in detail how the modifications introduced by this anomalous SC landscape in the DOS affect the optical conductivity, with the aim to understand how the emergence of several energy scales reflects in the quasiparticle dynamics probed by optical spectroscopy.

To compute fluctuations on top of the (inhomogeneous) BdG ground state, we evaluate dynamical correlation functions,

$$
\chi_{i j}(\hat{A}, \hat{B})=-i \int d t e^{i \omega t}\left\langle\mathcal{T} \hat{A}_{i}(t) \hat{B}_{j}(0)\right\rangle,
$$

where in the following $\hat{A}, \hat{B}$ correspond to either pair fluctuations, charge fluctuations, or current operators, i.e.,

$$
\begin{aligned}
\delta \Delta_{i} & \equiv c_{i \downarrow} c_{i \uparrow}-\left\langle c_{i \downarrow} c_{i \uparrow}\right\rangle, \\
\delta \Delta_{i}^{\dagger} & \equiv c_{i \uparrow}^{\dagger} c_{i \downarrow}^{\dagger}-\left\langle c_{i \uparrow}^{\dagger} c_{i \downarrow}^{\dagger}\right\rangle,
\end{aligned}
$$

$$
\begin{aligned}
\delta n_{i}^{\dagger} & \equiv \sum_{\sigma}\left(c_{i \sigma}^{\dagger} c_{i \sigma}-\left\langle c_{i \sigma}^{\dagger} c_{i \sigma}\right\rangle\right), \\
j_{i}^{\alpha} & =-i t \sum_{\sigma}\left[c_{i \sigma}^{\dagger} c_{i+\alpha, \sigma}-c_{i+\alpha, \sigma}^{\dagger} c_{i \sigma}\right],
\end{aligned}
$$

and expectation values are evaluated with the $\mathrm{BdG}$ ground state. To compute the optical conductivity one needs in particular the current-current correlation function. For a given disorder configuration the optical conductivity is given by

$$
\left.\sigma_{x x}(\omega)\right)=-e^{2} \frac{1}{N} \sum_{i j} \frac{-t_{i}^{x} \delta_{i j}-\chi_{i j}\left(\hat{j_{x}}, \hat{j_{x}}\right)}{i(\omega+i \delta)},
$$

where $t_{i}^{x}$ is the kinetic energy between sites $R_{i}$ and $R_{i+x}$. For definiteness we evaluate the response along the $x$ direction. The results shown in the following are then obtained by averaging over different disorder realizations. Note that we restrict here to the limit of zero temperature. While static thermal fluctuations within the BdG scheme have been investigated in Ref. [30], the additional inclusion of quantum fluctuations at finite temperature is technically challenging and will not be considered here.

As we discussed in the Introduction, the optical conductivity depends crucially on the level of approximation used to compute $\chi_{i j}\left(\hat{j}_{x}, \hat{j_{x}}\right)$. When the current response is evaluated at the level of the bare bubble we obtain the optical conductivity in the BCS approximation, with the inclusion of the effect of disorder on the quasiparticle excitations, described via the BdG transformation Eq. (2). The average over disorder restores the translational invariance of the system, but adds finite lifetimes to the energy levels in momentum space, in agreement with the general reasoning of the approach followed by Mattis and Bardeen [2], with the difference that we do not make any further assumption on the DOS in the normal or SC state. Once the vertex corrections are included all the contributions of the collective modes to the optical response are taken explicitly into account at RPA level, and the gauge-invariance of the theory is restored, as required for the $f$-sum rule to be fulfilled [37]. Further technical details can be found in Refs. [35,48].

\section{DENSITY OF STATES}

Even though the overall behavior of the SC DOS for the model Eq. (1) has been already discussed previously $[29,32]$, here we want to show more specifically how disorder modifies its fundamental shape both in the normal and SC state with respect to the homogeneous case. As we mentioned in the Introduction, tunneling experiments on disordered superconductors deviate in several respects from the usual BCS paradigm. First, a Altshuler-Aronov (AA) type correction to the DOS [55] is found, in the form of an extended dip around the Fermi level already at high temperatures [22,24]. Second, a pseudogap occurring at the energy scale of the SC gap survives above $T_{c}$, in a range of temperatures that increases for increasing disorder [21-25]. Figure 2 reveals a similar pseudogap feature in the normal state DOS, shown with a blue dashed line. In the present case, this gap is mainly caused by the unconstrained variation of the densities in the presence of an attractive onsite interaction. In fact, the local chemical potential at site $R_{i}$ is given by $E_{i}=V_{i}+U n_{i} / 2$ so 


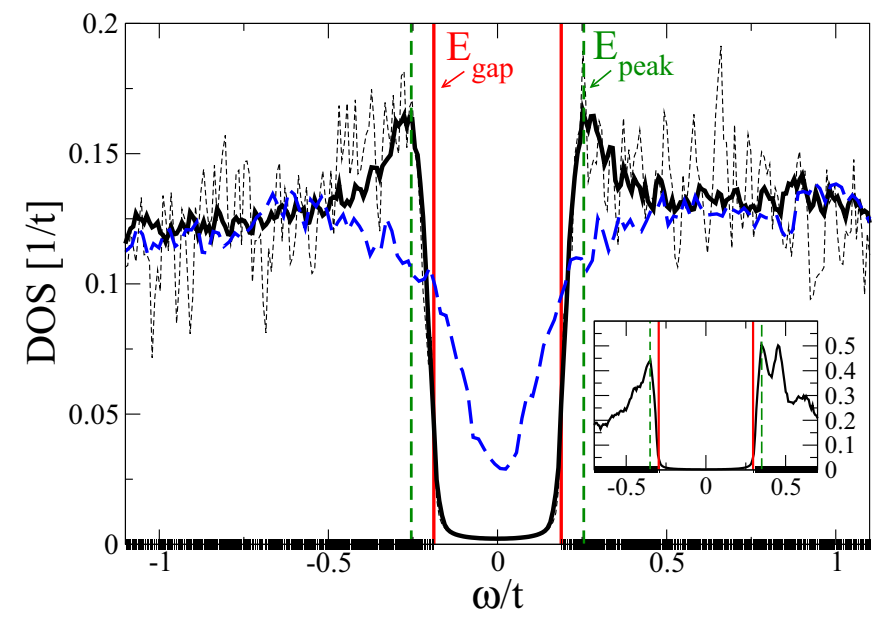

FIG. 2. Main panel: Density of states in the normal (blue) and superconducting state (black). The normal DOS is obtained from an average over 150 disorder configurations $\left(U / t=2, V_{0} / t=2\right)$ of $24 \times 24$ systems. The SC DOS (solid, black) is the average over 10 disorder configurations from $52 \times 52$ systems, whereas the black dashed curve is the DOS for a particular disorder realization. The eigenvalues of the latter system are indicated by bars on the frequency axis. Vertical lines indicate the position of the lowest eigenvalue (solid, red) and the "coherence" peak feature (dashed, green). Inset: same for a system with disorder in the local interaction $U_{i}=U_{0} \pm$ $\delta u_{i}$ with $U_{0} / t=2$ and $\delta u_{i}$ is taken from a flat distribution between $-0.5 t \leqslant \delta u_{i} \leqslant+0.5 t$. The higher energy peak of the double peak feature is a remainder of the van Hove singularity. In all cases the charge density is $n=0.875$.

that sites with $E_{i}$ just below (above) the Fermi energy tend to increase (lower) the local density to lower the total energy. This redistribution of charge is limited by the concomitant loss in kinetic energy and leads to the pseudogap in the normal state.

The black thick line in the main panel of Fig. 2 shows the DOS in the superconducting state, obtained as an average over 10 disorder configurations for large systems with $52 \times 52$ sites. The black thin dashed line is the DOS for a particular disorder realization. As can be seen both curves agree in the evolution of spectral weight from inside the gap toward the maximum DOS located at $E_{\text {peak }}$, which resemble coherence peak features. The eigenvalues of the $\mathrm{BdG}$ matrix (corresponding to the black dashed DOS) are shown as bars on the frequency axis. Clearly the DOS between the lowest (absolute) eigenvalue (i.e., the hard gap $E_{\text {gap }}$ ) and the "coherence peak" is determined by a large set of eigenvalues that induce a tail feature in the DOS. For this reason, the tail is also not related to the small $\eta=0.005 t$, which determines the width of the Lorentzians in our evaluation of the DOS. Thus, in contrast to the textbook profile of the BCS DOS, where the sharp coherence peaks mark also the onset of the hard gap, here two separate energy scales occur. In general, the hard gap $E_{\text {gap }}$ measuring the minimum energy required to create a single-particle excitation is lower than the value $E_{\text {peak }}$, where the spectral weight removed from low energy piles up giving rise to a smeared coherence peak. Notice that the separation between these two energy scales is similar to what has been discussed before in models with inhomogeneous distribution of the coupling constants $[14,56]$, based on the Larkin-Ovchinnikov original suggestion [43]. Indeed, the same result could be reproduced in our approach by introducing disorder in the local interaction $U_{i}$ instead of the local chemical potential. The result for $U_{i}=2 t \pm \delta u_{i}$, with $\delta u_{i}$ taken from a flat distribution between $-0.5 t \leqslant \delta u_{i} \leqslant+0.5 t \mathrm{a}$, is shown in the inset to Fig. 2. Clearly, the main features are similar to those of the main panel, namely a large set of eigenvalues that determines the smooth evolution of the DOS between the hard gap and the coherence peaks. However, as we shall see in the next section, the effects on the optical spectrum are completely different in the two classes of models. Indeed, when the separation between $E_{\text {peak }}$ and $E_{\text {gap }}$ is induced by in-gap bound states, as it happens for magnetic impurities or for inhomogeneous coupling constants, the BCS optical spectrum is only modified with respect to the MB one by the appearance of a tail below $2 E_{\text {peak }}[14,45,46]$. In contrast, for nonmagnetic impurities we find that the BCS response is only sensible to the scale $E_{\text {gap }}$, with an optical spectrum that resembles closely the $\mathrm{MB}$ one provided that the optical threshold is shifted down to $2 E_{\text {gap }}$.

\section{OPTICAL CONDUCTIVITY}

Once the modifications of the DOS in the normal and SC state due to an inhomogenous SC ground state have been established, let us now investigate how disorder affects the optical conductivity. Denoting by $\chi_{1,2}(\omega)$ the real and imaginary parts of the disorder average of $1 / N \sum_{n, m} \chi_{n m}\left(\hat{j}_{x}, \hat{j}_{x}\right)$ (i.e., the $q=0$ component), and by $\left\langle t_{x}\right\rangle$ the disorder average of the mean kinetic energy along the $x$ direction, one finds for the real and imaginary parts of $\sigma(\omega)$

$$
\begin{gathered}
\sigma_{1}(\omega)=\pi D_{s} \delta(\omega)-e^{2} \frac{\chi_{2}(\omega)}{\omega}, \\
\sigma_{2}(\omega)=e^{2} \frac{-\left\langle t_{x}\right\rangle+\chi_{1}(\omega)}{\omega},
\end{gathered}
$$

where the superfluid stiffness $D_{s}$ is given by $D_{s}=e^{2}\left[-\left\langle t_{x}\right\rangle+\right.$ $\chi_{1}(\omega=0)$ ]. Note that Fig. 3 only shows the regular part of $\sigma_{1}(\omega)$, i.e., without the superfluid $\delta$-like contribution of Eq. (9). It can be seen from Eq. (10) that the stiffness is also obtained as the limit $D_{s}=\lim _{\omega \rightarrow 0} \omega \sigma_{2}(\omega)$.

In a perfectly clean system the RPA current-current correlation function at finite frequency vanishes, so that $D_{s}$ equals the so-called diamagnetic term $D_{d} \equiv-e^{2}\left\langle t_{x}\right\rangle$, which coincides with $n e^{2} / m$ in a system with parabolic band dispersion. In this case, $\sigma_{1}(\omega)=\pi D_{s} \delta(\omega)$ and $\sigma_{2}(\omega)=D_{s} / \omega$ [57]. Disorder changes this picture in two ways. First, similar to what happens in the normal state, single-particle excitations acquire a finite lifetime broadening $\Gamma$ in momentum space, making finite-frequency absorption possible, as encoded in a finite value of $\chi_{2}(\omega)$ in Eq. (9). Since by Kramers-Kronig $\chi_{1}(0)=$ $\int d \omega \chi_{2}(\omega) / \omega$ the finite-frequency absorption results in a reduction of the stiffness $D_{s}$ with respect to the diamagnetic term $D_{d}$. When $\chi(\omega)$ is computed at BCS level one obtains the suppression of $D_{s}$ due to MB theory of the order of $D_{s} \sim D_{d}(\Delta / \Gamma)$. Notice that the optical sum rule is always satisfied, since the total integrated spectral weight,

$$
\int_{0}^{\infty} d \omega \sigma_{1}(\omega)=\pi D_{d},
$$



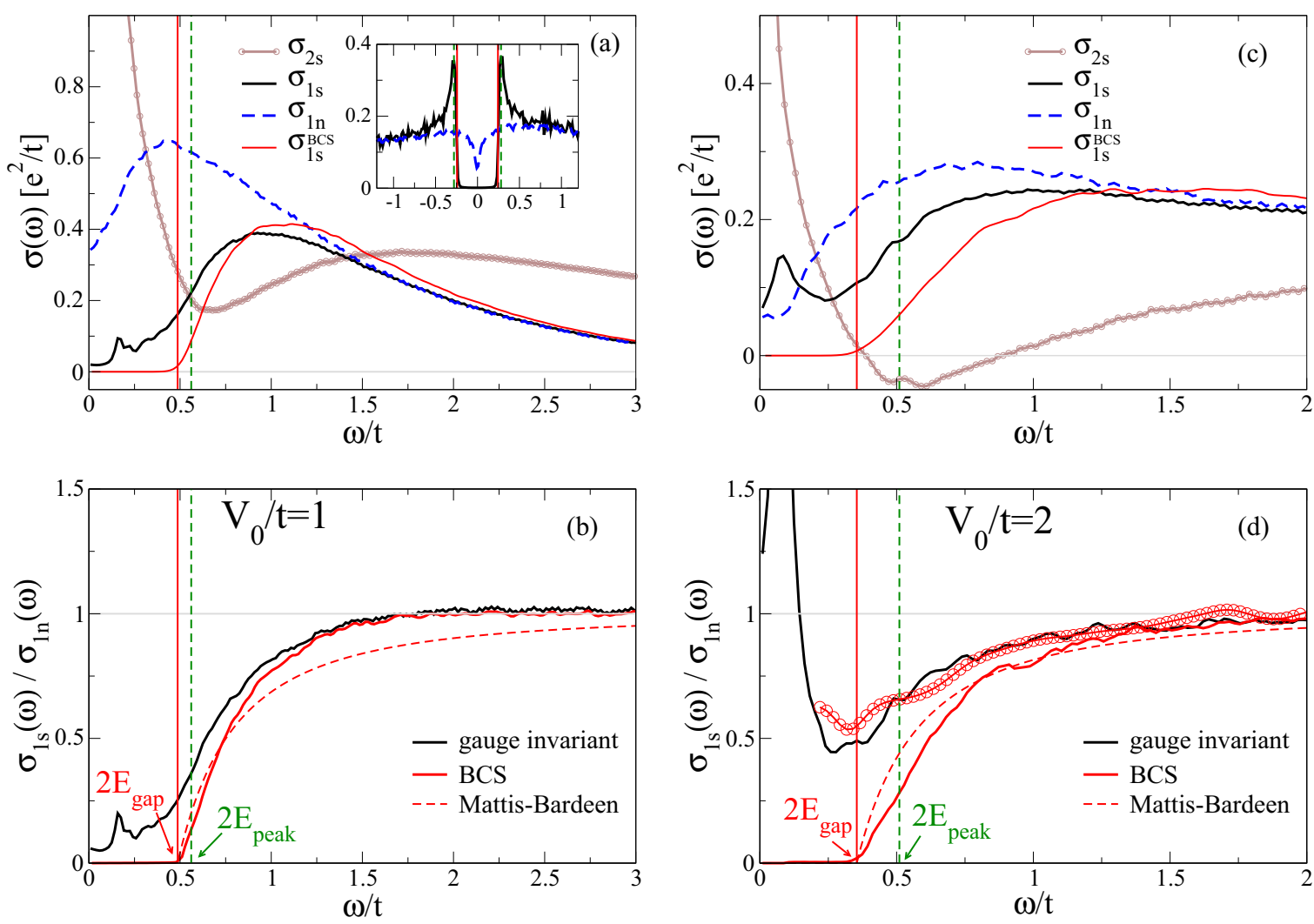

FIG. 3. Optical conductivity at $V_{0} / t=1$ (panels a, b) and at $V_{0} / t=2$ (right panels $\mathrm{c}, \mathrm{d}$ ). The remaining parameters are: $U / t=2$, $n=0.875,24 \times 24$ lattice. Panels (a) and (c) show the real (solid, black) and imaginary (dotted, maroon) part of the optical conductivity in the SC state and $\sigma_{1}$ in the normal state (dashed, blue) evaluated as an average over 50 disorder configurations within the gauge-invariant RPA approach. For comparison, the real part of the conductivity in the SC state within the BCS approximation is also shown by the red solid line. The vertical green and red lines denote $2 E_{\text {peak }}$ and $2 E_{\text {gap }}$, respectively, as extracted from the DOS. The DOS for the case $V_{0} / t=2$ is the one shown in Fig. (2), while the one for $V_{0} / t=1$ is shown explicitly as an inset of panel (a). Panels (b) and (d) show the ratio between the real part of the optical conductivity computed in the SC and normal state. The red curve reports the corresponding result in the BCS limit and the dashed line is the Mattis-Bardeen fit done using $2 E_{\text {gap }}$ as the optical threshold. For comparison, panel (d) also shows experimental data from Ref. [14] for the most disordered sample $\left(\mathrm{T}_{c}=3.8 \mathrm{~K}\right)$ and we have scaled the energy by $t=0.8 \mathrm{THz}$. Notice that for the BCS case also $\sigma_{1, n}$ has been computed at the bare-bubble level, leading to a normal-state conductivity slightly different from the gauge-invariant result reported in the upper panels.

still scales with $D_{d}$. Second, once that collective modes are included via the vertex corrections one expects a further reduction of $D_{s}$, as it has been pointed out already in Ref. [33] by direct computation of the stiffness within a gauge-invariant approach. In particular, it turned out that $D_{s}$ can be reduced by orders of magnitude with respect to its BCS counterpart in the presence of strong disorder. As a consequence the imaginary part of $\sigma(\omega)$ can even become negative at small energies, a feature that is also observed experimentally [14] for the strongly disordered samples. In addition, the suppression of the stiffness will be compensated, due to the optical sum rule, by an extra absorption with respect to the BCS or MB bare-bubble estimate.

All these features are correctly captured by the BCS and the full gauge-invariant RPA calculations of the optical conductivity shown in Fig. 3 for two values of disorder. In the upper panels we report the absolute values of $\sigma_{1}$ and $\sigma_{2}$ in the $\mathrm{SC}$ state, along with $\sigma_{1}$ in the normal state. As one can see, already at intermediate disorder $\sigma_{1}(\omega)$ shows a suppression at low-energy in the normal state, that is specular to the analogous suppression of the DOS discussed in Fig. 2 (see also the DOS at $V_{0} / t=1$ shown in the inset of Fig. 3). When one enters the SC state $\sigma_{1}(\omega)$ is further suppressed due to the opening of a full SC gap. The optical spectral weight is then transferred to the delta peak at zero frequency (not shown), and manifests in a finite imaginary response $\sigma_{2}(\omega)$ that diverges as $D_{s} / \omega$ at low frequencies.

To establish a closer connection with the usual MB results we report in the lower panels of Fig. 3 the ratio between $\sigma_{1, s / n}$ in the SC and normal state, in the case of a BCS (red line) or full gauge invariant (black line) calculation. Notice that despite the strong inhomogeneity of the underlying SC state [29,32,35] the BCS ratio $\sigma_{1, s} / \sigma_{1 n}$ shows a hard threshold developing at twice the quasiparticle excitation gap $E_{\text {gap }}$, and not at twice the coherence-peak scale $E_{\text {peak }}$, as it has been sometimes argued in the analysis of the experimental data [13]. This is particularly evident in Fig. 3(d) where the separation between the two scales is larger. The persistence of a hard gap in the BCS response suggests to compare the $\mathrm{BCS}$ calculations with the outcomes of the standard MB formula, with $\Delta$ replaced by $E_{\text {gap }}$. As can be seen in Figs. 3(b) and 3(d) the overall trend of the BCS result is captured by the standard MB formula, 
even though the MB fit overestimates the absorption close to the onset, whereas for large $\omega$ it stays slightly below the BCS curve. As we will discuss in more details in the next section, these discrepancies can be attributed to the assumption of a constant normal-state DOS, implicit in the MB approach.

When the contribution of the collective modes is considered the extra optical absorption becomes finite even below $2 E_{\text {gap }}$, with an intensity and shape which depend on the disorder level. At intermediate disorder, Fig. 3(b), the collective modes are responsible for the emergence of a tail below the quasiparticle optical gap, that strongly resembles the recent measurements of Ref. [14]. In this respect, our results share analogies and differences with respect to the interpretation proposed recently in Ref. [14]. Here the authors argue that the existence of quasiparticle states between the true optical gap, i.e., $2 E_{\text {gap }}$, and the scale $2 E_{\text {peak }}$ identified by the maximum in the DOS, can be captured by means of an inhomogeneity-induced smearing of the conventional $\mathrm{MB}$ response starting at $2 E_{\text {peak }}$. The inhomogeneous MB fit in Ref. [14] is derived from a distribution of the coupling constant [43] so that $2 E_{\text {peak }}$ is still a meaningful scale related to the average SC gap. Our results, which instead are derived from local onsite disorder, confirm the identification of the optical gap with $2 E_{\text {gap }}$, but they also show that the scale $2 E_{\text {peak }}$ does not play any role in the BCS response, which displays a $\mathrm{MB}$-like sharp optical onset at $2 E_{\text {gap }}$. The extra absorption below the $2 E_{\text {gap }}$ threshold (which from an experimental perspective hampers the identification of this energy scale [14]) is due instead to collective modes, whose contribution can even become visible well above it at stronger disorder; see Fig. 3(d). As we shall see below, our view is further supported by the direct comparison of the BCS calculations with experimental data in samples at intermediate disorder, that can be very well reproduced within a modified MB scheme with the only exception of a small subgap tail.

\section{EFFECT OF A PSEUDOGAP IN THE MATTIS-BARDEEN FORMULA}

The MB theory [2] starts from the Pippard's expression describing the nonlocal relation between the current density $\mathbf{j}(\mathbf{r}, t)$ found in response to an applied frequency dependent vector potential $\mathbf{A}\left(\mathbf{r}^{\prime}, \omega\right)$. To account for disorder, the idea is to multiply the BCS kernel $K\left(\mathbf{r}-\mathbf{r}^{\prime}, \omega\right)$, relating these two quantities, with a factor $\exp \left[-\left|\mathbf{r}-\mathbf{r}^{\prime}\right| /(2 l)\right]$, where $l$ is the mean-free path. For strong disorder and in the Pippard's limit $\lambda \ll \xi_{0}$ this simplifies the integration over $\mathbf{r}^{\prime}$, leading to a rather simple expression for the complex optical conductivity. Later on it has been shown by Nam [7] that the same result holds also in the London limit $\left(\lambda \gg \xi_{0}\right)$ as far as the impurity scattering is weakly frequency dependent. Nonetheless, in both cases one assumes that the DOS in the normal state is constant over the energy scale of the SC gap, giving a constant $\sigma_{1}$ above $T_{c}$. However, as discussed in the previous sections, the DOS of a strongly disordered superconductor is expected to develop a pseudogap already in the normal state. This feature, that is reproduced by our results already at the level of the BCS approximation, has been also observed in the tunneling spectra of several systems [22,24-26]. More specifically, a systematic investigation of $\mathrm{NbN}$ samples [24] has shown a normal-state V-shaped background in the DOS that extends up to high bias. This nearly temperature-independent broad background persists up to temperatures well above $T_{c}$ and it has been attributed to Altshuler-Aronov-type electron-electron interactions. In addition to this smooth suppression the samples of higher disorder level show also a SC-like pseudogap, identified by its occurrence on the range of energy where the SC gap develops below $T_{c}$ and up to temperatures $T^{*} \sim 8 \mathrm{~K}$. This is the same disorder level where a pseudogaplike behavior has been recently reported in the conductivity[14], as a suppression of $\sigma(\omega)$ on the scale of the SC gap which is already visible above $T_{c}$.

To reproduce the pseudogap features of our BCS results and of the experimental data we rederived the MB formula by explicitly keeping any energy dependence of the normal-state DOS $N(\omega)$ on the scale of the SC gap. In the following, we will also assume a DOS symmetric around the chemical potential $N(\omega)=N(-\omega)$, while the general result is given by Eq. (A1) in Appendix A. For the symmetric case, one finds

$$
\begin{gathered}
\frac{\sigma_{1 s}}{\sigma_{1 n}}=\frac{I(\omega)}{\Gamma(\omega)}, \\
\Gamma(\omega)=\frac{1}{\omega} \int_{-\infty}^{\infty} d \epsilon N(\epsilon) N(\epsilon+\omega)[f(\epsilon)-f(\epsilon+\omega)], \\
I(\omega)=\frac{2}{\omega} \int_{\Delta}^{\infty} d E \frac{f(E)-f(E+\omega)}{\epsilon_{1} \epsilon_{2}} \\
\times\left[E(E+\omega)+\Delta^{2}\right] N\left(\epsilon_{1}\right) N\left(\epsilon_{2}\right) \\
-\frac{\Theta(\omega-2 \Delta)}{\omega} \int_{\Delta-\omega}^{-\Delta} d E \frac{1-2 f(E+\omega)}{\varepsilon_{1} \varepsilon_{2}} \\
\times\left[E(E+\omega)+\Delta^{2}\right] N\left(\epsilon_{1}\right) N\left(\epsilon_{2}\right), \\
\epsilon_{1}=\sqrt{E^{2}-\Delta^{2}}, \\
\epsilon_{2}=\sqrt{(E+\omega)^{2}-\Delta^{2}},
\end{gathered}
$$

where the functions $\Gamma(\omega)$ and $I(\omega)$ correspond, up to a constant, to the conductivities in the normal and SC state, respectively. In fact, $\Gamma(\omega)$ approaches a constant for a constant DOS $N(\omega)=$ const., as it is the case for the normal-state conductivity in the standard MB theory, where the broadening of the Drude peak occurs on the scale of a scattering rate much larger than $\Delta$. We emphasize once more that the modifications of the usual MB formula encoded in Eq. (12)-(16) are completely different with respect to the ones discussed in previous work $[14,45,46]$. Indeed, the goal of Refs. [14,45,46] is to modify the superconducting DOS appearing in the MB formula to reproduce the emergence of the two energy scales $E_{\text {peak }}$ and $E_{\text {gap }}$. In contrast, our goal is to investigate how the anomalies of the normal-state DOS reflect on the BCS response, which has been already shown in the previous section to be sensitive only to the scale set in by $E_{\text {gap }}$.

Concerning the imaginary part of $\sigma(\omega)$ the "standard" way in deriving this quantity is via a continuation of the squareroot functions in the complex plane, i.e., in the quantity $\epsilon_{2}$ in Eq. (16). However, this procedure in the present case is quite cumbersome since $\epsilon_{2}$ also appears in the frequency-dependent DOS. Thus, we find it more convenient to calculate $\sigma_{2}(\omega)$ via a Kramers-Kronig transformation of $\sigma_{1}(\omega)$ as given by Eq. (12). To account also for the $\delta$ peak of $\sigma_{1}(\omega)$ at zero frequency 


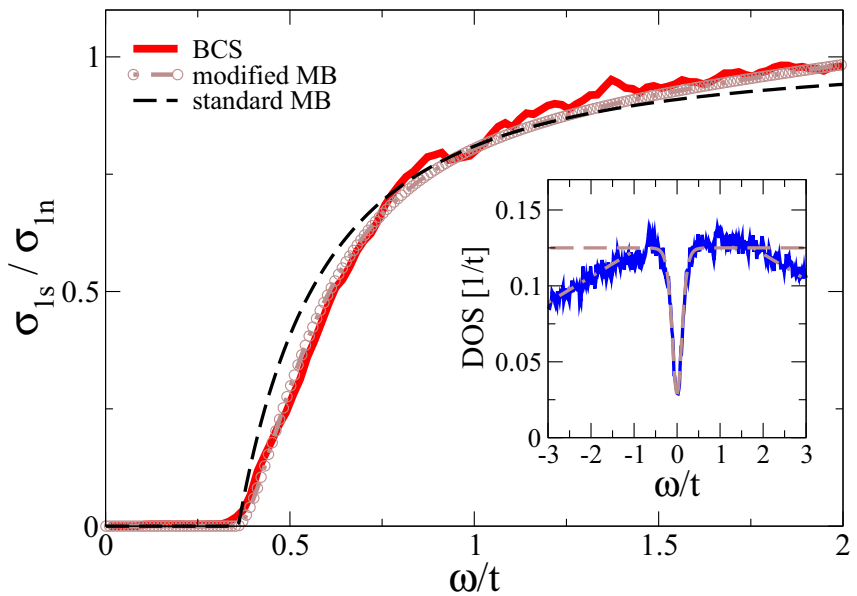

FIG. 4. Ratio between optical conductivities in the superconducting and normal state for the Hubbard model at $V_{0} / t=2$ [as in Figs. 2 and $3(\mathrm{~d})]$ in the BCS approximation (red line), compared to the prediction of the standard MB theory (dashed black line) and of the modified MB formula Eq. (12) (brown symbols). Inset: fit of the numerical results for the normal-state DOS of the Hubbard model. Near the Fermi level we used the approximated formula Eq. (20) with $\alpha=0.0156 / t, \beta=0.1095 / t, \Omega=0.1968 t$, while away from it we used a linear extrapolation at large energies.

[see Eq. (9)], we add to $\sigma_{2}$ a $1 / \omega$ term whose strength can be obtained from the standard MB expression for $\sigma_{2}(\omega)$ in the limit $\omega \rightarrow 0$ [see Eq. (A3)], where the DOS at the chemical potential $N(0)$ can be taken in front of the integral,

$$
\begin{aligned}
\lim _{\omega \rightarrow 0} \frac{\omega \sigma_{2}(\omega)}{\sigma_{1 n}}= & \frac{n_{s} e^{2}}{m \sigma_{1 n}} \\
= & \frac{N^{2}(0)}{\Gamma(0)} \lim _{\omega \rightarrow 0} \int_{\Delta-\omega}^{\Delta} d E[1-2 f(E+\omega)] \\
& \times \frac{E(E+\omega)+\Delta^{2}}{\sqrt{\Delta^{2}-E^{2}} \sqrt{(E+\omega)^{2}-\Delta^{2}}} \\
= & \pi \Delta
\end{aligned}
$$

and the last equality holds in the limit of zero temperature.

To test the validity of Eq. (12), we first compare it with the results obtained for the optical conductivity at the BCS level, shown by the red solid line in Figs. 3(b) and 3(d). To model the energy-dependent DOS we use the following approximation for the normal-state DOS:

$$
N(\omega) / N(\infty)=\alpha+\beta \tanh ^{2}\left(\frac{\omega}{\Omega}\right) .
$$

As one can see in the inset to Fig. 4, Eq. (20) yields a very good approximation to the disorder averaged BdG DOS around the chemical potential. However, since the latter deviates from a bare constant at larger energies, we have in addition also implemented a DOS linear in frequency beyond the pseudogap, as shown by the dashed-dotted lines in the inset to Fig. 4 .

The modified absorption due to the presence of an energydependent DOS is compared with standard MB theory in the main panel of Fig. 4. As one can see, accounting properly for the energy dependence of the normal-state excitations yields an excellent description of the BCS results within the modified MB scheme Eq. (12). First, the DOS suppression near the Fermi level allows us to correctly capture the onset of absorption close to the hard gap of the DOS, which is overestimated within the standard MB formula. At larger frequency the linear trend observed in the DOS reflects in a saturation of the BCS result faster than the standard $\mathrm{MB}$ prediction at $\omega / t \approx 1$, which is again well captured by our modified MB scheme.

\section{COMPARISON WITH THE EXPERIMENTS}

Once clarified, the role of the inhomogeneous SC background and of the pseudgap on the BCS and full GI optical conductivity let us now compare our results with the experimental data from Ref. [14], where a systematic investigation of the role of disorder in several $\mathrm{NbN}$ samples has been provided. We first focus on a sample in the intermediate disorder regime $\left(T_{c}=\right.$ $6.2 \mathrm{~K}$ ), where one finds a pseudogap behavior in the normal state, evidenced by a depression of $\sigma_{1 n}$ at low frequencies [see Fig. 5(a)], but without the emergence of significant subgap features. According to the previous discussion, we expect that in this regime the optical conductivity can be well reproduced by the BCS calculation. To account also for the effects of an energy-dependent DOS we then apply the modified MB formula Eq. (12). We determine the parameters entering the DOS Eq. (20) by minimizing the differences between experimental and theoretical $\sigma_{1}(\omega)$ [from Eq. (12)] in the normal and superconducting state, respectively. The resulting DOS is reported in the lower right inset to Fig. 5(a) with a depletion at the chemical potential of $\beta /(\alpha+\beta)=22 \%$ and width $\Omega=0.58 \mathrm{THz}$. Figure 5 demonstrates that for the real part of for $\sigma(\omega)$ this approach leads to an excellent agreement with experimental data both in the superconducting ( $T=1.45 \mathrm{~K})$ and normal $(T=7 \mathrm{~K})$ state, respectively. On the other hand, standard Mattis-Bardeen theory with a constant DOS (dashed line in Fig. 5) overestimates the recovery of spectral weight in the superconducting state and would of course predict a constant $\sigma_{1 n}$ in the normal state.

The fit for the imaginary part $\sigma_{2}(\omega)$ is shown in Fig. 5(b). Below $T_{c}$ the contribution arising from the $1 / \omega$ part, calculated from Eq. (19), depends sensitively on the value of the DOS at the chemical potential $N(0)$. Since the experiment strongly loses signal below $\sim 0.3 \mathrm{THz}$, it is difficult to estimate this value from the optical data. Despite this uncertainty the fit from our extended Mattis-Bardeen theory gives a good account of the data, both below $T_{c}$ and in the normal state. In Figs. 5(a) and 5(b), we also show the effect of taking the ratio $\sigma_{1 s} / \sigma_{1 n}$ in analyzing the data. As one can see, while for the real part the errors in the standard MB formula cancel out each other in the fit of $\sigma_{1 s} / \sigma_{1 n}$, for the imaginary part the usual MB approach fails completely to recover the frequency dependence of $\sigma_{2 s} / \sigma_{1 n}$ above the optical gap. The analysis for a second, less disordered, sample is reported in Appendix B.

At stronger disorder the microwave absorption deviates substantially from the $\mathrm{MB}$, and even our modified $\mathrm{MB}$ scheme cannot reproduce the experiments, since the effect of collective modes should be included. In this regime our model system Eq. (1) does not have enough flexibility to reproduce separately both the normal-state pseudogap of the DOS and the anomalous subgap absorption below $T_{c}$. Nonetheless, by 

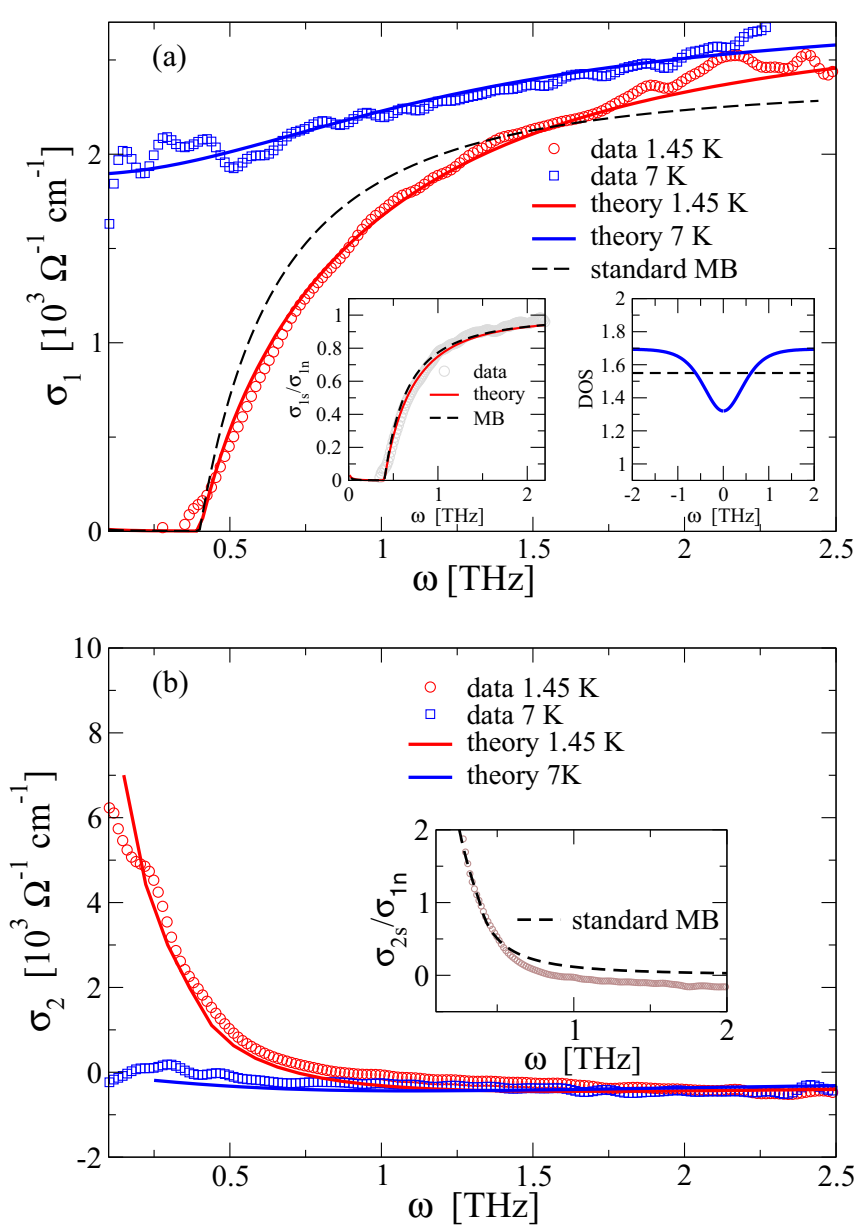

FIG. 5. Real (a) and imaginary (b) part of the optical conductivity for a NbN sample with $T_{c}=6.2 \mathrm{~K}$. Data at $T=7 \mathrm{~K}$ and $T=1.45 \mathrm{~K}$ are shown by squares (blue) and circles (red); the corresponding fits from the modified MB theory Eq. (12) are the solid lines, while the dashed black line is the standard MB formula with a constant DOS. In both cases we used $\Delta=0.2 \mathrm{THz}$. The left inset of panel (a) and the inset (b) shows the ratio between the $T=1.45$ and $T=7 \mathrm{~K}$ data (symbols) compared with conventional and modified MattisBardeen theory. Notice that while in the ratio $\sigma_{1 s} / \sigma_{1 n}$ the effect of the DOS disappears, making the standard MB formula apparently good, the ratio $\sigma 2 s / \sigma 1 n$ cannot be well reproduced by the standard MB approach. The right inset of panel shows the DOS used to reproduce the normal-state conductivity, with parameters $\alpha=1.32, \beta=0.37$, $\Omega=0.58 \mathrm{THz}$ in Eq. (20). Experimental data by courtesy of the authors of Ref. [14].

tuning only the value of the hopping, which fixes the energy units in the Hubbard model, we can give a good account for the behavior of $\sigma_{1 s} / \sigma_{1 n}$, as shown in Fig. 3(d). This result is particularly striking, considering the lack of long-range repulsive Coulomb interactions in our model which may modify the low frequency part of the spectrum. Indeed, as it has been discussed in Ref. [48], the extra spectral weight at strong disorder is due mainly to the contribution of phase fluctuations, which become optically active due to the presence of disorder $[48,50,58]$. Since in our model the phase mode is a sound, the extra absorption manifests mainly at low frequencies. In real systems one could expect that long-range Coulomb
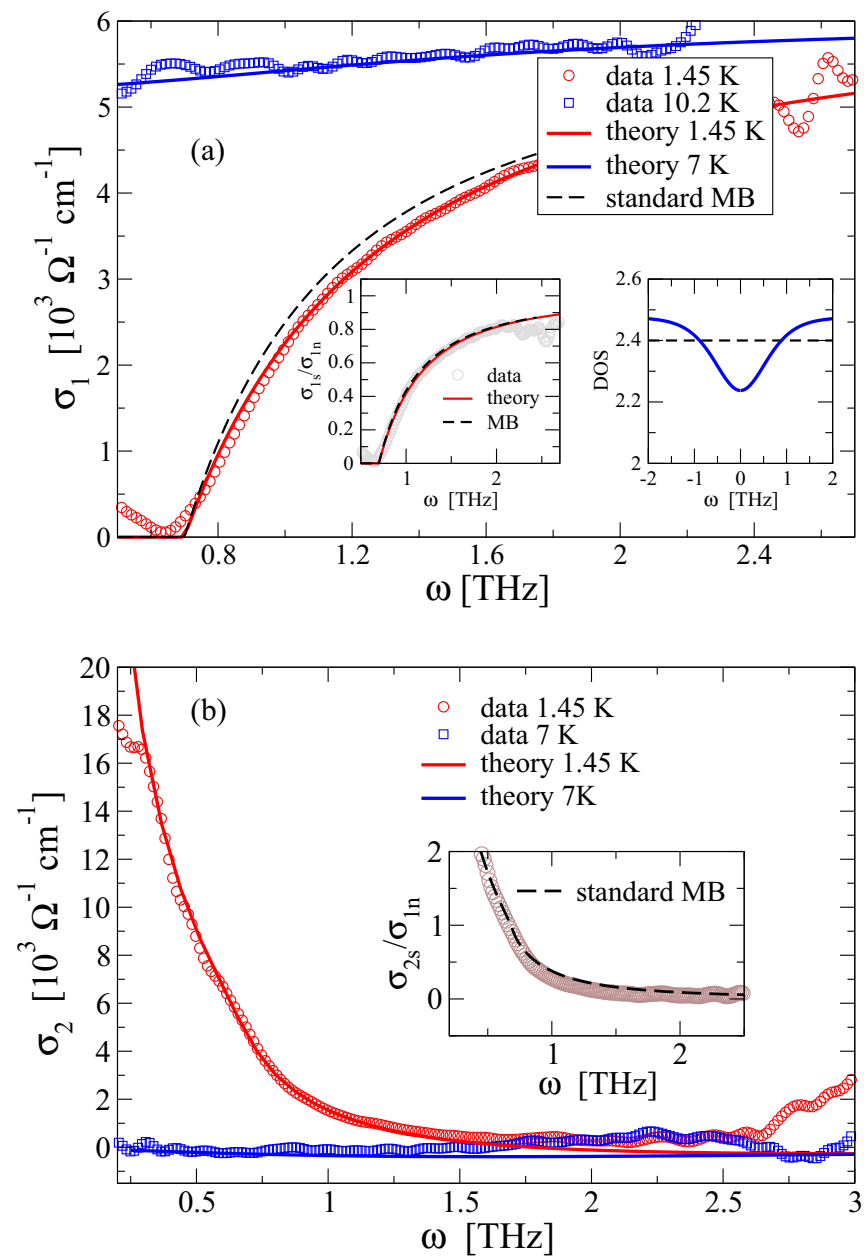

FIG. 6. Same as Fig. 5 but for a NbN sample with $T_{c}=9.2 \mathrm{~K}$. Parameters for the DOS Eq. (20): $\alpha=2.24, \beta=0.24, \Omega=0.75 \mathrm{THz}$. Superconducting gap parameter: $\Delta=0.35 \mathrm{THz}$. Experimental data by courtesy of the authors of Ref. [14].

interactions push at least in part this extra absorption to higher energy [59]. However, the surprisingly good qualitative and quantitative agreement between our full GI calculations and the experiments shown in Fig. 3 suggests that this effect is not so severe. We expect that this is due both to the small value of the stiffness and to the strong inhomogeneity induced by disorder, that makes the optically-active phase modes rather local and therefore less sensitive to the Coulomb interaction.

\section{CONCLUSIONS}

In this work we analyzed several key aspects which must be taken into account to correctly interpret the optical measurements in disordered SC films from weak to strong disorder, to account for significant deviations from the conventional MB theory. As we discussed in detail, in general the optical response can be computed in the bare-bubble or BCS approximation, that corresponds to include only the effects of quasiparticle excitations, or beyond that by adding the effect of collective modes via vertex corrections. The MB theory corresponds to the BCS approximation in the presence of disorder, included via the introduction of a finite length 
scale setting the correlation between two plane-wave states. As a consequence, within the MB approach disorder does not influence the DOS, which is a constant in the normal state and reduces to the usual clean BCS form in the SC state, with two sharp coherence peaks at the gap value and zero available state below it. In our paper we have critically analyzed these assumptions by evaluating the optical response for systems where disorder is implemented by a random on-site potential, and the optical response is computed both in the BCS approximation and by including vertex corrections at RPA level.

We first analyzed the BCS optical response, by making a direct connection with the average DOS of our disordered system, which shows itself significant deviations with respect to the clean case. Indeed, the self-organized formation of SC islands induced by disorder suppresses considerably the sharp coherence peaks at $E_{\text {peak }}$ and generates tails extending down to a lower hard gap $E_{\text {gap. }}$. This effect was already known to occur for inhomogeneities in the SC coupling parameter[43,56]. However, our model of disorder seems more appropriate to describe the experimental observation[25] that the formation of nanoscale SC islands in the disordered films is not related to the atomic scale inhomogeneity, which would also induce a related "atomic scale" modulation of the coupling constant. Despite the existence of two separate energy scales $E_{\text {gap }}<E_{\text {peak }}$ in the DOS, the BCS response still displays a hard optical absorption at $2 E_{\text {gap }}$. This effect can be well captured by the MB formula provided that this energy scale, and not $2 E_{\text {peak }}$ as usually assumed [13,14], is identified with the parameter $2 \Delta$ in the MB formula. We have shown (cf. Figs. 5 and 6) that this identification strongly improves the analysis of the optical data for less disordered samples within MB theory. Further improvement can be achieved by implementing the presence of a pseudogap feature in the normal-state DOS, i.e., a DOS frequency-dependent on the scale of $E_{\text {peak }}$ (cf. Figs. 5 and 6). We have shown that this effect can be included within the MB scheme via the formulas Eqs. (12)-(16), that could be helpful for experimental data analysis, allowing for a consistent fit of both $\sigma_{1 n}$ and $\sigma_{1 s}$ separately. In fact, as shown in Fig. 6, a MB fit of $\sigma_{1 s} / \sigma_{1 n}$ alone can artificially yield a reasonable fit although $\sigma_{1 n}$ displays strong variations at low energy. The consistent procedure suggested by our analysis would be to first extract the frequency-dependent DOS via a fit of the normal-state optical conductivity and then to implement it in the fit of optical data in the SC state, which then provides the value of $2 E_{\text {gap }}$.

While at weak disorder the BCS-based MB theory correctly describes the optical conductivity of the system, this approximation fails at stronger disorder, i.e., sizable $V_{0} / t$ in the model Eq. (1), since collective modes become optically active. We have shown that their inclusion within a gaugeinvariant RPA scheme removes the sharp onset of absorption at $2 E_{\text {gap }}$, building up additional spectral weight below the quasiparticle contribution. From an experimental point of view this hampers the determination of $2 E_{\text {gap }}$ which in some cases may be still extracted from an extrapolation of an extended MB fit [Eqs. (12)-(16)] and a concomitant analysis of the tunneling data. As discussed in Ref. [48], the largest contribution below the spectral gap is due to the absorption of phase modes, with an admixture of amplitude and charge excitations. Thus, one would expect that exact shape and strength of the extra absorption could be affected by the inclusion of long-range Coulomb forces. Nonetheless, the main trends of the experiments are already captured by our calculations, see Fig. 3, suggesting that screening effects are probably not so dramatic in real systems. If this is just a consequence of the low value of the plasma mode, due to the low superfluid density, or it is an inherent effect of the local character of the screened phase modes at strong disorder is an interesting open question that we leave for future investigation.

\section{ACKNOWLEDGMENTS}

We acknowledge useful discussions with P. Armitage, and we thank the authors of Ref. [14] for providing us with the experimental data shown in the figures. This work has been supported by the Italian MIUR under Projects FIRB-HybridNanoDev-RBFR1236VV, PRINRIDEIRON2012X3YFZ2, Premiali-2012 ABNANOTECH and by the Italian MAECI under the Italian-India collaborative project SUPERTOP-PGR0487. G.S. acknowledges support from the Deutsche Forschungsgemeinschaft.

\section{APPENDIX A: MATTIS-BARDEEN FORMULA FOR FREQUENCY DEPENDENT DOS}

The formula Eq. (12) for the regular part $\sigma_{1}(\omega)$ given in the main text is valid for a symmetric DOS $N(\omega)$ around the chemical potential. The general formula reads

$$
\begin{aligned}
I(\omega)= & \frac{1}{2 \omega} \int_{\Delta}^{\infty} d E \frac{f(E)-f(E+\omega)}{\epsilon_{1} \epsilon_{2}}\left\{\left[E(E+\omega)+\epsilon_{1} \epsilon_{2}+\Delta^{2}\right]\left[N\left(\epsilon_{1}\right) N\left(\epsilon_{2}\right)+N\left(-\epsilon_{1}\right) N\left(-\epsilon_{2}\right)\right]\right. \\
& +\left[E(E+\omega)-\epsilon_{1} \epsilon_{2}+\Delta^{2}\right]\left[N\left(\epsilon_{1}\right) N\left(-\epsilon_{2}\right)+N\left(\left(-\epsilon_{1}\right) N\left(\epsilon_{2}\right)\right]\right\} \\
& -\frac{1}{4 \omega} \int_{\Delta-\omega}^{-\Delta} d E \frac{1-2 f(E+\omega)}{\varepsilon_{1} \varepsilon_{2}}\left\{[ E ( E + \omega ) + \epsilon _ { 1 } \epsilon _ { 2 } + \Delta ^ { 2 } ] \left[N\left(\epsilon_{1}\right) N\left(\epsilon_{2}\right)+N\left(\left(-\epsilon_{1}\right) N\left(-\epsilon_{2}\right)\right]\right.\right. \\
& \left.+\left[E(E+\omega)-\epsilon_{1} \epsilon_{2}+\Delta^{2}\right]\left[N\left(\epsilon_{1}\right) N\left(-\epsilon_{2}\right)+N\left(-\epsilon_{1}\right) N\left(\epsilon_{2}\right)\right]\right\} .
\end{aligned}
$$


As one can immediately see, when $N(\omega)=N_{0}=$ const., one recovers back the standard MB formula. In particular in the limit $T \rightarrow 0$ one recovers from Eq. (13) that $\Gamma(\omega)=N_{0}^{2}$. By computing also $I(\omega)$, one finds

$$
\begin{aligned}
\frac{\sigma_{1 s}}{\sigma_{1 n}}= & \frac{2}{\omega} \int_{\Delta}^{\infty} d E[f(E)-f(E+\omega)] \frac{\left[E(E+\omega)+\Delta^{2}\right]}{\sqrt{E^{2}-\Delta^{2}} \sqrt{(E+\omega)^{2}-\Delta^{2}}} \\
& -\frac{\Theta(\omega-2 \Delta)}{\omega} \int_{\Delta-\omega}^{-\Delta} d E[1-2 f(E+\omega)] \frac{\left[E(E+\omega)+\Delta^{2}\right]}{\sqrt{E^{2}-\Delta^{2}} \sqrt{(E+\omega)^{2}-\Delta^{2}}} \\
\frac{\sigma_{2 s}}{\sigma_{1 n}} & =\frac{2}{\omega} \int_{-\Delta, \Delta-\omega}^{\infty} d E[1-2 f(E+\omega)] \frac{\left[E(E+\omega)+\Delta^{2}\right]}{\sqrt{\Delta^{2}-E^{2}} \sqrt{(E+\omega)^{2}-\Delta^{2}}},
\end{aligned}
$$

where together with the real part we also wrote explicitly the imaginary part of the conductivity for the standard MB case. In the expression for $\sigma_{2}$ the lower limit of integration is taken as the largest between the two values $-\Delta$ and $\Delta-\omega$.

\section{APPENDIX B: ANALYSIS OF $T_{c}=9.2 \mathrm{~K}$ DATA}

Figure 6 shows the fits from extended Mattis-Bardeen theory for a disordered $T_{c}=9.2 \mathrm{~K}$ sample. The analysis of the data is given in Sec. $\mathrm{V}$ of the main text.

[1] J. Bardeen, L. N. Cooper, and J. R. Schrieffer, Phys. Rev. 106, 162 (1957); 108, 1175 (1957).

[2] D. C. Mattis and J. Bardeen, Phys. Rev. 111, 412 (1958).

[3] A. A. Abrikosov and L. P. Gor'kov, Zh. Eksp. Teor. Fiz. 35, 1558 (1958) [Sov. Phys. JETP 8, 1090 (1959)].

[4] W. Zimmermann, E. H. Brandt, M. Bauer, E. Seider, and L. Genzel, Physica C 183, 99 (1991).

[5] N. M. Rugheimer, A. Lehoczky, and C. V. Briscoe, Phys. Rev. 154, 414 (1967).

[6] D. M. Ginsberg and L. C. Hebel, in Nonequilibrium Properties: Comparison of Experimental Results with Predictions of the BCS Theory in Superconductivity, editied by R. D. Parks (Marcel Dekker, Inc., New York, 1969), Vol. I.

[7] S. B. Nam, Phys. Rev. 156, 470 (1967); 156, 487 (1967).

[8] L. H. Palmer and M. Tinkham, Phys. Rev. 165, 588 (1968).

[9] K. Steinberg, M. Scheffler, and M. Dressel, Phys. Rev. B 77, 214517 (2008).

[10] R. W. Crane, N. P. Armitage, A. Johansson, G. Sambandamurthy, D. Shahar, and G. Grüner, Phys. Rev. B 75, 094506 (2007).

[11] E. F. C. Driessen, P. C. J. J. Coumou, R. R. Tromp, P. J. de Visser, and T. M. Klapwijk, Phys. Rev. Lett. 109, 107003 (2012).

[12] P. C. J. J. Coumou, E. F. C. Driessen, J. Bueno, C. Chapelier, and T. M. Klapwijk, Phys. Rev. B 88, 180505 (2013).

[13] D. Sherman, U. S. Pracht, B. Gorshunov, S. Poran, J. Jesudasan, M. Chand, P. Raychaudhuri, M. Swanson, N. Trivedi, A. Auerbach, M. Scheffler, A. Frydman, and M. Dressel, Nat. Phys. 11, 188 (2015).

[14] B. Cheng, L. Wu, N. J. Laurita, H. Singh, M. Chand, P. Raychaudhuri, and N. P. Armitage, Phys. Rev. B 93, 180511 (2016).

[15] M. Žemlička, P. Neilinger, M. Trgala, M. Rehák, D. Manca, M. Grajcar, P. Szabó, P. Samuely, Š. Gaži, U. Hübner, V. M. Vinokur, and E. Il'ichev, Phys. Rev. B 92, 224506 (2015).

[16] J. Simmendinger, U. S. Pracht, L. Daschke, T. Proslier, J. A. Klug, M. Dressel, and M. Scheffler, Phys. Rev. B 94, 064506 (2016).

[17] N. Bachar, U. Pracht, E. Farber, M. Dressel, G. Deutscher, and M. Scheffler, J. Low Temp. Phys. 179, 83 (2014).
[18] U. S. Pracht, N. Bachar, L. Benfatto, G. Deutscher, E. Farber, M. Dressel, and M. Scheffler, Phys. Rev. B 93, 100503 (2016); U. S. Pracht, T. Cea, N. Bachar, G. Deutscher, E. Farber, M. Dressel, M. Scheffler, C. Castellani, A. M. Garcí-Garacía, and L. Benfatto, ibid. 96, 094514 (2017).

[19] J. Corson, J. Orenstein, S. Oh, J. O’Donnell, and J. N. Eckstein, Phys. Rev. Lett. 85, 2569 (2000).

[20] B. Sacépé, C. Chapelier, T. I. Baturina, V. M. Vinokur, M. R. Baklanov, and M. Sanquer, Phys. Rev. Lett. 101, 157006 (2008).

[21] B. Sacépé, C. Chapelier, T. I. Baturina, V. M. Vinokur, M. R. Baklanov, and M. Sanquer, Nat. Commu. 1, 140 (2010).

[22] M. Mondal, A. Kamlapure, M. Chand, G. Saraswat, S. Kumar, J. Jesudasan, L. Benfatto, V. Tripathi, and P. Raychaudhuri, Phys. Rev. Lett. 106, 047001 (2011).

[23] B. Sacépé, T. Dubouchet, C. Chapelier, M. Sanquer, M. Ovadia, D. Shahar, M. Feigel'man, and L. Ioffe, Nat. Phys. 7, 239 (2011).

[24] M. Chand, G. Saraswat, A. Kamlapure, M. Mondal, S. Kumar, J. Jesudasan, V. Bagwe, L. Benfatto, V. Tripathi, and P. Raychaudhuri, Phys. Rev. B 85, 014508 (2012).

[25] A. Kamlapure, T. Das, S. C. Ganguli, J. B. Parmar, S. Bhattacharyya, and P. Raychaudhuri, Sci. Rep. 3, 2979 (2013).

[26] Y. Noat, V. Cherkez, C. Brun, T. Cren, C. Carbillet, F. Debontridder, K. Ilin, M. Siegel, A. Semenov, H.-W. Hübers, and D. Roditchev, Phys. Rev. B 88, 014503 (2013).

[27] P. Szabó, T. Samuely, V. Hašková, J. Kačmarčík, M. Žemlička, M. Grajcar, J. G. Rodrigo, and P. Samuely, Phys. Rev. B 93, 014505 (2016).

[28] G. Lemarie, A. Kamlapure, D. Bucheli, L. Benfatto, J. Lorenzana, G. Seibold, S. C. Ganguli, P. Raychaudhuri, and C. Castellani, Phys. Rev. B 87, 184509 (2013).

[29] A. Ghosal, M. Randeria, and N. Trivedi, Phys. Rev. B 65, 014501 (2001).

[30] Y. Dubi, Y. Meir, and Y. Avishai, Nature 449, 876 (2007).

[31] L. B. Ioffe and M. Mezard, Phys. Rev. Lett. 105, 037001 (2010); M. V. Feigelman, L. B. Ioffe, and M. Mezard, Phys. Rev. B 82, 184534 (2010).

[32] K. Bouadim, Y. L. Loh, M. Randeria, and N. Trivedi, Nat. Phys. 7, 884 (2011). 
[33] G. Seibold, L. Benfatto, C. Castellani, and J. Lorenzana, Phys. Rev. Lett. 108, 207004 (2012).

[34] S. Ghosh and S. S. Mandal, Phys. Rev. Lett. 111, 207004 (2013).

[35] G. Seibold, L. Benfatto, C. Castellani, and J. Lorenzana, Phys. Rev. B 92, 064512 (2015).

[36] R. C. Dynes, V. Narayanamurti, and J. P. Garno, Phys. Rev. Lett. 41, 1509 (1978); R. C. Dynes, J. P. Garno, G. B. Hertel, and T. P. Orlando, ibid. 53, 2437 (1984).

[37] J. R. Schrieffer, Theory of Superconductivity (Addison-Wesley, Reading, MA, 1988).

[38] S. Weinberg, The Quantum Theory of Fields: Modern Applications (Cambridge University Press, Cambridge, England, 1996), Vol. 2.

[39] N. Nagaosa, Quantum Field Theory in Condensed Matter Physics (Springer-Verlag, Berlin, Heidelberg, New York, 1999).

[40] S. De Palo, C. Castellani, C. Di Castro, and B. K. Chakraverty, Phys. Rev. B 60, 564 (1999).

[41] A. Paramekanti, M. Randeria, T. V. Ramakrishnan, and S. S. Mandal, Phys. Rev. B 62, 6786 (2000).

[42] L. Benfatto, A. Toschi, and S. Caprara, Phys. Rev. B. 69, 184510 (2004).

[43] A. I. Larkin and Yu. N. Ovchinnikov, Zh. Eksp. Teor. Fiz. 61, 2147 (1971)

[44] M. A. Skvortsov and M. V. Feigel'man, JETP 117, 487 (2013).

[45] K. Maki, in Superconductivity, edited by R. D. Parks (Marcel Dekker, New York, 1969), Vol. 2.

[46] Ya. V. Fominov, M. Houzet, and L. I. Glazman, Phys. Rev. B 84, 224517 (2011).
[47] Y. V. Fominov and M. A Skvortsov, Phys. Rev. B 93, 144511 (2016).

[48] T. Cea, D. Bucheli, G. Seibold, L. Benfatto, J. Lorenzana, and C. Castellani, Phys. Rev. B 89, 174506 (2014).

[49] D. Podolsky, A. Auerbach, and D. P. Arovas, Phys. Rev. B 84, 174522 (2011).

[50] M. Swanson, Y. L. Loh, M. Randeria, and N. Trivedi, Phys. Rev. X 4, 021007 (2014).

[51] See e.g. D. Pekker and C. M. Varma, Annu. Rev. Condens. Matter Phys. 6, 269 (2015) and references therein.

[52] T. Cea, C. Castellani, G. Seibold, and L. Benfatto, Phys. Rev. Lett. 115, 157002 (2015).

[53] V. F. Gantmakher and V. T. Dolgopolov, Phys. Usp. 53, 1 (2010).

[54] M. V. Feigel'man, L. B. Ioffe, V. E. Kravtsov, and E. Cuevas, Ann. Phys. 325, 1390 (2010).

[55] Electron-electron Interactions in Disordered Systems, edited by B. L. Altshuer, A. G. Aronov, A. L. Efros, and M. Pollak (Elsevier, North Holland, 1985).

[56] M. V. Feigel'man and M. A. Skvortsov, Phys. Rev. Lett. 109, 147002 (2012).

[57] It must be noticed that for the interacting model on the lattice $\sigma_{1}(\omega)$ computed beyond RPA is finite at finite frequency even in the clean case; see, e.g., Douglas J. Scalapino, Steven R. White, and Shoucheng Zhang, Phys. Rev. B 47, 7995 (1993).

[58] S. Barabash, D. Stroud, and I.-J. Hwang, Phys. Rev. B 61, R14924 (2000); S. V. Barabash and D. Stroud, ibid. 67, 144506 (2003).

[59] P. W. Anderson, Phys. Rev. 130, 439 (1962). 